$$
\begin{gathered}
\text { SF } 291 \\
B 7
\end{gathered}
$$





\section{Horse Breeders' and Trainers' Comparison Table}

Copyright, 1915

2w by

J. W. BROWN 


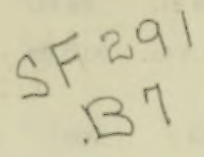

$4290 \quad 4620 \quad 4950 \quad 5280 \quad 5610 \quad 5940 \quad 6270 \quad 660$ )

$\begin{array}{llllllll}\frac{13}{16} & 7 / 8 & \frac{1}{16} & 1 \mathrm{M} & 1 \frac{1}{7} & 11 / 8 & 1 \frac{3}{16} & 11 / 4\end{array}$

$\begin{array}{llll}65 & 70 & 75 & 80\end{array}$

$\begin{array}{llll}105 & 110 & 115 & 120\end{array}$

$\begin{array}{llll}67 & 72\} & 78 & 83\}\end{array}$

$107\} \quad 112$ \& $118 \quad 123$ !

$\begin{array}{llll}70\} & 75\} & 81 & 86\}\end{array}$

$\left.\left.110 \frac{1}{6} 115\right\} \quad 121 \quad 126\right\}$

$\begin{array}{llll}721 & 78\} & 84 & 89\end{array}$

$\begin{array}{llll}112 \xi & 118\} & 124 & 129 ?\end{array}$

$\begin{array}{llll}75\} & 81\} & 87 & 92\}\end{array}$

$\begin{array}{llll}115\} & 121\} & 127 & 132\}\end{array}$

$\begin{array}{llllllll}78 & 84 & 90 & 96 & 102 & 108 & 114 & 120\end{array}$

$\begin{array}{llllllll}118 & 124 & 130 & 136 & 142 & 148 & 154 & 2 \mathrm{M}\end{array}$

$\begin{array}{llllllll}80\} & 86 & 93 & 99\} & 105\} & 111\} & 117\} & 124\end{array}$

$\begin{array}{lllllllll}120\} & 126\} & 133 & 139\} & 145\} & 151\} & 157\} & 204\end{array}$

$\begin{array}{llllllll}83\} & 89\} & 96 & 102\} & 108\} & 115\} & 121\} & 128\end{array}$

$\begin{array}{llllllll}123\} & 129\} & 136 & 142\} & 148\} & 155\} & 201\} & 208\end{array}$

$\begin{array}{llllllll}85\} & 92\} & 99 & 105\} & 112\} & 118\} & 125\} & 132\end{array}$

$\begin{array}{llllllll}125 & 132\} & 139 & 145\} & 152\} & 158\} & 205\} & 212\end{array}$

$\begin{array}{llllllll}88\} & 95\} & 102 & 108\} & 115\} & 122\} & 129\} & 136\end{array}$

$\begin{array}{llllllll}128\} & 135 \frac{\xi}{3} & 142 & 148\} & 155\} & 202\} & 209\} & 216\end{array}$

$\begin{array}{llllllll}91 & 98 & 105 & 112 & 119 & 126 & 133 & 140\end{array}$

$\begin{array}{llllllll}131 & 138 & 145 & 152 & 159 & 206 & 213 & 220\end{array}$

$\begin{array}{llllllll}93\} & 100\} & 108 & 115\} & 122\} & 129\} & 136\} & 144\end{array}$

$\begin{array}{llllllll}133\} & 140 \text { \& } & 148 & 155 ! & 202\} & 209 ! & 216 ! & 224\end{array}$

$\begin{array}{llllllll}96\} & 103\} & 111 & 118\} & 125\} & 133\} & 140\} & 148\end{array}$

$\begin{array}{lllllllll}136\} & 143\} & 151 & 158\} & 205\} & 213 \xi & 220\} & 228\end{array}$

$\begin{array}{llllllll}98\{ & 106\} & 114 & 121\} & 129\} & 136\} & 144\} & 152\end{array}$

$\begin{array}{llllllll}138\{ & 146\} & 154 & 201\} & 209\} & 216\} & 224\} & 232\end{array}$

$\begin{array}{llllllll}101\} & 109\} & 117 & 124 ! & 132\} & 140\} & 148\} & 156\end{array}$

$\begin{array}{llllllll}141\} & 149\} & 157 & 204\} & 212 ! & 220\} & 228 ! & 236\end{array}$

$\begin{array}{llllllll}104 & 112 & 120 & 128 & 136 & 144 & 152 & 160\end{array}$

$\begin{array}{llllllll}144 & 152 & 2 \mathrm{Min} & 208 & 216 & 224 & 232 & 240\end{array}$

$\begin{array}{llllllll}106 ! & 114 ! & 123 & 131\} & 139\} & 147\} & 155\} & 164\end{array}$

$\begin{array}{llllllll}146\} & 154\} & 203 & 211\} & 219\} & 227\} & 235\} & 244\end{array}$

$\begin{array}{llllllll}109\} & 117\} & 126 & 134\} & 142 ! & 151\} & 159\} & 168\end{array}$

$\begin{array}{llllllll}149\} & 157\} & 206 & 214\} & 222\} & 231\} & 239\} & 248\end{array}$

$\begin{array}{llllllll}111\} & 120\} & 129 & 137\} & 146\} & 154\} & 163\} & 172\end{array}$

$\begin{array}{llllllll}151\} & 200\} & 209 & 217\} & 226\} & 234\} & 243\} & 252\end{array}$

$\begin{array}{llllllll}114\} & 123 & 132 & 140\} & 149\} & 158\} & 167 \mid & 176\end{array}$

$\begin{array}{llllllll}154\} & 203\} & 212 & 220\} & 229\} & 238\} & 247\} & 256\end{array}$

$\begin{array}{llllllll}117 & 126 & 135 & 144 & 153 & 162 & 171 & 180\end{array}$

$\begin{array}{llllllll}157 & 203 & 215 & 224 & 233 & 242 & 251 & 3\end{array}$ 


\section{$\begin{array}{llllllllllllllllllllllll}690 & 7260 & 7590 & 7920 & 8250 & 8580 & 8910 & 9240 & 9570 & 9900 & 10230 & 10560 & 10890 & 11220 & 11550 & 11880 & 12210 & 12540 & 12870 & 13200\end{array}$}

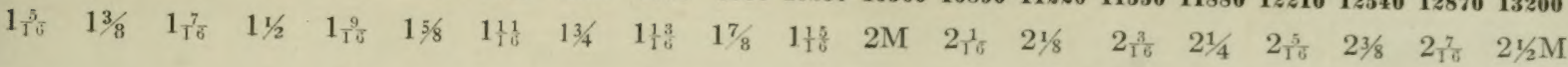

12 in. $=1$ foot

$3 \mathrm{ft}$. 1 yard

$5 \frac{1}{2} \mathrm{yds} .=1 \mathrm{rod}=16 ! \mathrm{ft}$.

40 rods $=1$ furlong $=660 \mathrm{ft}$.

$8 \mathrm{fur} .=1 \mathrm{mile}=5280 \mathrm{ft}$.

\begin{tabular}{|c|c|c|c|c|c|c|c|c|c|c|c|c|c|c|c|c|c|c|c|}
\hline $\begin{array}{l}126 \\
206\end{array}$ & $\begin{array}{l}132 \\
212\end{array}$ & $\begin{array}{l}138 \\
218\end{array}$ & $\begin{array}{l}144 \\
224\end{array}$ & 150 & 156 & 162 & 163 & 174 & 180 & 186 & 192 & 193 & 231 & 210 & 216 & 222 & 228 & 234 & 240 \\
\hline 206 & 212 & 218 & 224 & 230 & 236 & 242 & 248 & 254 & $3 \mathrm{Min}$ & 306 & 312 & 318 & 324 & 330 & 336 & 342 & 348 & 354 & $4 \mathrm{Min}$ \\
\hline $130 \frac{1}{5}$ & $136 \frac{2}{2}$ & $1423^{3}$ & $148 \frac{4}{5}$ & 155 & $161 \frac{3}{5}$ & $167 \frac{2}{5}$ & $173_{5}^{3}$ & $179 \frac{4}{5}$ & 186 & $192 \frac{1}{3}$ & $198 \frac{2}{5}$ & $204 \frac{3}{3}$ & $210 \frac{4}{5}$ & 217 & $223 \frac{1}{3}$ & $229 \frac{2}{5}$ & $235 \frac{3}{3}$ & $241 \frac{1}{3}$ & 248 \\
\hline $210 \frac{1}{3}$ & $216_{\frac{1}{s}}^{2}$ & $222 \frac{3}{5}$ & $223 \frac{4}{5}$ & 235 & $241 \frac{1}{3}$ & $247 \frac{2}{5}$ & $253 \frac{3}{5}$ & $259 \frac{4}{3}$ & 306 & $312 \frac{1}{5}$ & $318 \frac{2}{5}$ & $324 \frac{3}{3}$ & $330 \frac{4}{3}$ & 337 & $343 \frac{1}{s}$ & $349 \frac{2}{3}$ & $355 ?$ & $401 \frac{1}{3}$ & 408 \\
\hline $134 \frac{2}{5}$ & $140 \frac{4}{5}$ & $147 \frac{1}{3}$ & $153 \frac{3}{3}$ & 160 & $166 ?$ & $172 \frac{4}{3}$ & $179 \frac{1}{3}$ & $185 \frac{3}{5}$ & 192 & $193 \frac{2}{5}$ & 2043 & $211 \frac{1}{5}$ & $217 \frac{3}{5}$ & 224 & $230 \frac{2}{3}$ & 235 है & $243 \frac{1}{5}$ & $249 \frac{3}{3}$ & 256 \\
\hline $214 \frac{2}{3}$ & $220 \frac{4}{5}$ & $227 \frac{1}{3}$ & $233 \frac{3}{5}$ & 240 & $246 \frac{2}{3}$ & $252 \frac{4}{3}$ & $259 \frac{1}{5}$ & $305_{5}^{3}$ & 312 & $318 \frac{2}{5}$ & 324 & $331 \frac{1}{5}$ & $337 \frac{3}{5}$ & 344 & $350 \frac{2}{5}$ & $356\}$ & $403 \frac{1}{3}$ & $409\}$ & 416 \\
\hline $\begin{array}{l}138 ? \\
218 ?\end{array}$ & $\begin{array}{l}145 \frac{1}{5} \\
225 \frac{1}{4}\end{array}$ & $151 \frac{4}{5}$ & $158 \frac{2}{3}$ & 165 & $171 \frac{3}{5}$ & $178 \frac{1}{5}$ & $184 \frac{4}{5}$ & $191 \frac{2}{3}$ & 198 & 2043 & $211 \frac{1}{5}$ & $217 \frac{4}{5}$ & $224 \frac{2}{3}$ & 231 & $237 \frac{3}{5}$ & $244\}$ & $250 \frac{\xi}{3}$ & $257 \xi$ & 264 \\
\hline $218_{\mathrm{s}}^{3}$ & $225 \frac{1}{5}$ & $231 \frac{4}{5}$ & $238 \frac{2}{3}$ & 245 & $251 \frac{3}{3}$ & $258 \frac{1}{5}$ & $304 \frac{4}{5}$ & $311 \frac{2}{5}$ & 318 & $324 \frac{3}{5}$ & $331 \frac{1}{5}$ & $337 \frac{4}{5}$ & $344 ?$ & 351 & $357 \frac{3}{3}$ & $404 \frac{1}{5}$ & 410 s & $417 \frac{2}{3}$ & 424 \\
\hline $142 \frac{4}{5}$ & 1493 & $156 \frac{2}{5}$ & $163 \frac{1}{5}$ & 170 & $176_{3}^{4}$ & $183 \frac{3}{5}$ & $190 \frac{2}{5}$ & $197 \frac{1}{3}$ & 204 & 210 년 & $217 \frac{3}{5}$ & $224_{5}^{2}$ & 231 & 238 & $244 \frac{4}{5}$ & $251 \frac{3}{5}$ & $259 \frac{3}{3}$ & $265 \frac{1}{3}$ & 272 \\
\hline $222 \frac{1}{3}$ & $229_{5}^{\frac{3}{5}}$ & $236 \frac{2}{5}$ & $243 \frac{1}{5}$ & 250 & $256 \frac{4}{5}$ & $303_{5}^{3}$ & $310_{\mathrm{s}}^{2}$ & $317 \frac{1}{5}$ & 324 & $330_{5}^{4}$ & $337 \frac{3}{5}$ & $344 \frac{2}{5}$ & $351 \frac{1}{5}$ & 358 & $404 \frac{4}{5}$ & $411 \frac{3}{5}$ & $418 \frac{2}{5}$ & $425 \frac{1}{3}$ & 432 \\
\hline
\end{tabular}

\begin{tabular}{|c|c|c|c|c|c|c|c|c|c|c|c|c|c|c|c|c|c|c|c|}
\hline 907 & 154 & 161 & 168 & 175 & 182 & 189 & 196 & 203 & 210 & 217 & 224 & 231 & 238 & 245 & 252 & 259 & 266 & 273 & \\
\hline 227 & 234 & 241 & 248 & $25 \overline{5}$ & 302 & 309 & 316 & 323 & 330 & 337 & 344 & 351 & 358 & 405 & 412 & & 426 & 433 & 440 \\
\hline & $58 \frac{2}{5}$ & & $172 \frac{4}{5}$ & & & & & & & & & 237 & $244 \frac{4}{5}$ & 252 & $259 \frac{1}{5}$ & $266 \frac{2}{3}$ & 273 ? & $280 \mathrm{~s}$ & 288 \\
\hline & $238 \frac{2}{5}$ & $245 \frac{3}{5}$ & $252 \xi$ & $3 \mathrm{Min}$ & $307 \frac{1}{5}$ & $314 \frac{2}{5}$ & $321 \frac{3}{5}$ & $328 \frac{4}{5}$ & 336 & $43 \frac{1}{5}$ & & $357 \frac{3}{5}$ & & 412 & & & & & 48 \\
\hline & $162 \frac{4}{5}$ & & $177 \frac{3}{5}$ & 185 & $192 \frac{2}{3}$ & & & & 222 & $29 \frac{2}{5}$ & 236 & $244 \frac{1}{3}$ & $251 \frac{3}{3}$ & 259 & $266 \frac{2}{5}$ & $\frac{4}{5}$ & $281 \frac{1}{3}$ & $288 \frac{3}{3}$ & 296 \\
\hline $8:$ & $242 \frac{1}{5}$ & $250 \frac{1}{5}$ & $257 \frac{3}{5}$ & 305 & 3125 & 319 & $327 \frac{1}{5}$ & 534 है & 342 & $349 \frac{2}{5}$ & 356 & $404 \frac{1}{5}$ & & 419 & & & $441 \frac{1}{5}$ & $448 \frac{3}{5}$ & 56 \\
\hline & $67 \frac{1}{5}$ & $174 \frac{4}{5}$ & $82 ?$ & 190 & $97 \frac{\pi}{5}$ & & \& & 20 & 8 & $\frac{3}{3}$ & & 250 & 258 & 266 & 273 한 & & $288 \frac{4}{3}$ & $296\}$ & 304 \\
\hline 35 & 47 & 254 & $302 \frac{3}{5}$ & 310 & 3175 & 325 & 332 & $340^{\frac{2}{5}}$ & 348 & 3553 & $405 \frac{3}{3}$ & $410_{\frac{4}{5}}^{\frac{4}{3}}$ & & 426 & & $441 \frac{1}{3}$ & & $456 \frac{2}{s}$ & 04 \\
\hline & $71 \frac{3}{5}$ & $170^{2}$ & $187 \frac{1}{3}$ & & & & & & & & & & & 27 & & & & & 12 \\
\hline & 51 & 259 & $307 \frac{1}{3}$ & 315 & 322 & 330 & $338 \frac{2}{5}$ & $346 \frac{1}{3}$ & 354 & $401 \frac{1}{5}$ & 409 & $417 \frac{2}{s}$ & $425 \frac{1}{3}$ & 433 & 0 安 & & & $504\}$ & \\
\hline & & & & & & & & & & & 25 & & & & & & & & 320 \\
\hline & 256 & 304 & 312 & 320 & 328 & 336 & 344 & 352 & 4Min & 408 & 416 & 424 & 432 & 440 & 448 & 456 & & 512 & \\
\hline & $180^{\frac{2}{5}}$ & $188 \frac{3}{3}$ & $196 \frac{1}{5}$ & & & & & & & & & & & 28 & & & & & \\
\hline & $300 \frac{2}{s}$ & $308 \frac{3}{5}$ & 316 宩 & 325 & $333 \frac{1}{5}$ & & $349 \frac{3}{5}$ & $357 \frac{4}{8}$ & 406 & $114 \frac{1}{5}$ & $422 \frac{2}{5}$ & $430 \frac{2}{3}$ & & 447 & & & & 5194 & \\
\hline & 84 s & $193 \frac{1}{6}$ & $201 \frac{3}{5}$ & & & & & & 25 & & & 27 & & & & & & & \\
\hline & 304 है & $313\}$ & $321 \frac{3}{5}$ & 330 & $338 \frac{2}{5}$ & & $355 \frac{1}{s}$ & $403 \frac{3}{5}$ & 412 & $420^{?}$ & $428 \frac{1}{3}$ & $437 \frac{1}{5}$ & & 454 & 502 & & & & \\
\hline & $189 \frac{1}{3}$ & $197 \frac{4}{5}$ & $06 \frac{2}{3}$ & & & & & & & & & & & & & & & & \\
\hline & $309 \frac{1}{3}$ & $317 \frac{1}{3}$ & $326 ?$ & 335 & $343 \frac{3}{5}$ & $352 \frac{1}{3}$ & $400 \frac{4}{3}$ & $409 \frac{3}{3}$ & 418 & $426 ?$ & $435 \frac{1}{5}$ & 445 & & 501 & 50 & & & & \\
\hline & $193 \frac{3}{5}$ & $202 \frac{2}{3}$ & $211\}$ & 220 & 289 & & & & & & & & & & & & & & \\
\hline & & $322 \frac{2}{3}$ & $331 \frac{1}{3}$ & $3 ; 0$ & $348\}$ & & 400 z & 41 & 424 & 321 & $441 ?$ & & & 508 & 51 & & & & \\
\hline & & & & & & & & & & & & & & & & & 34 & & \\
\hline & & 327 & 336 & 345 & 354 & 403 & 412 & 421 & 430 & 453 & 448 & 457 & 506 & 515 & 524 & 533 & 542 & 551 & \\
\hline
\end{tabular}




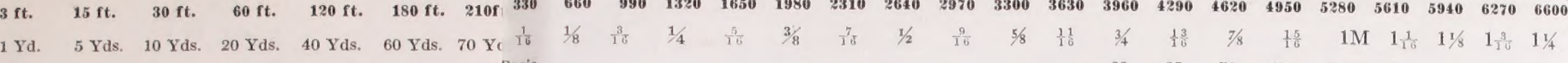

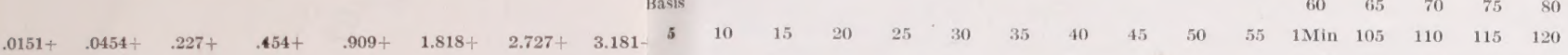

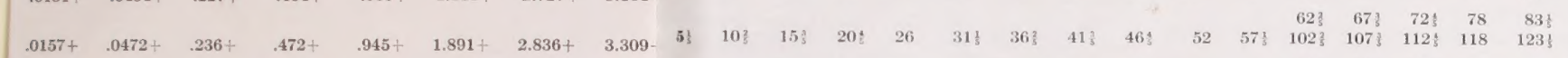

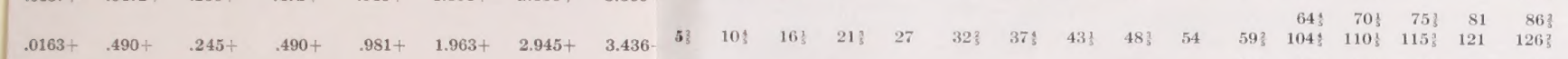
$\begin{array}{lllllllllllllllllllllll}6013 & 673 & 724 & 783 & 84 & 893 \\ 1293\end{array}$

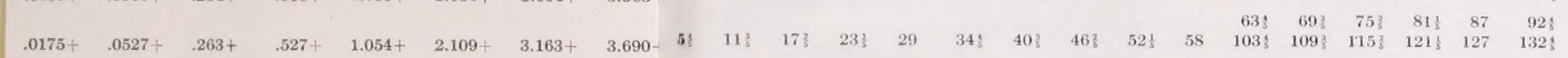

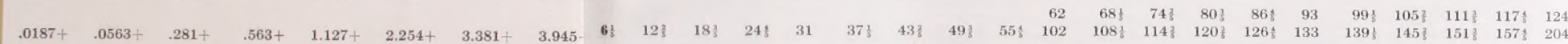

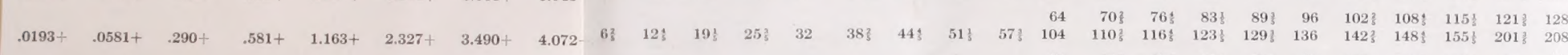

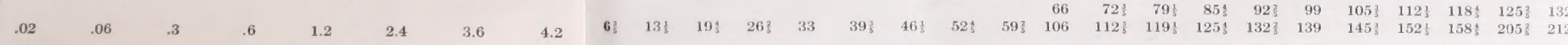

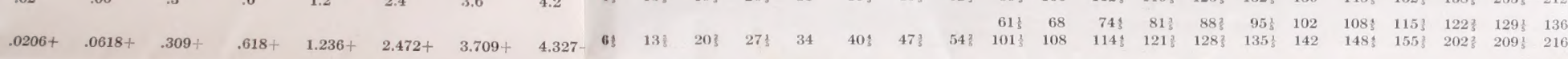

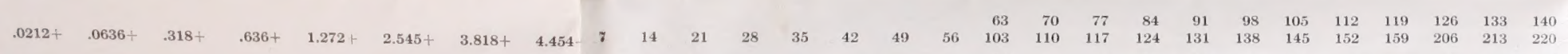

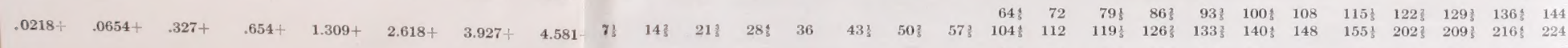

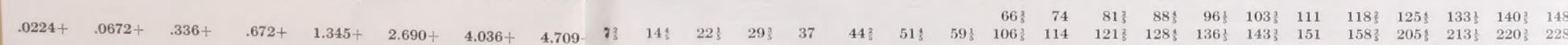

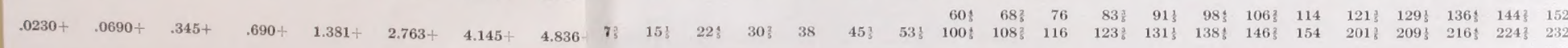

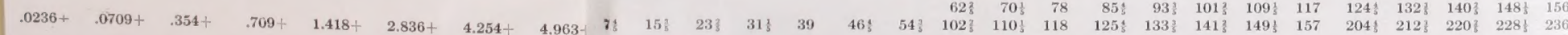

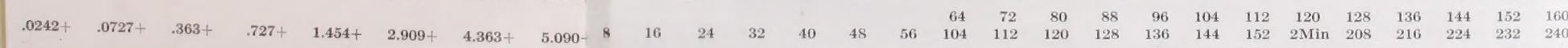

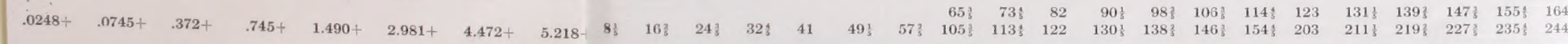

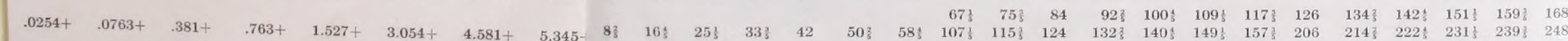

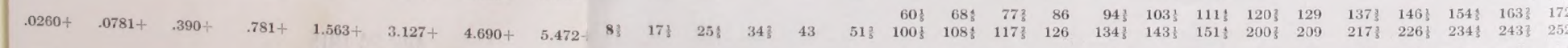

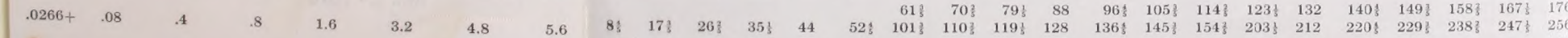

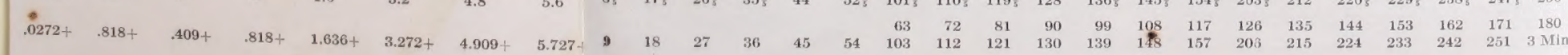

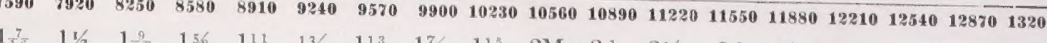

\section{2 in. $=1$ foot
$3 \mathrm{ft}=1$ yard

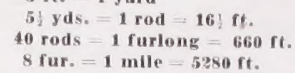

$\begin{array}{llllllllllllllllllll}1266 & 132 & 138 & 144 & 150 & 156 & 162 & 163 & 174 & 180 & 186 & 192 & 193 & 234 & 210 & 216 & 222 & 228 & 234 & 240 \\ 206 & 212 & 218 & 224 & 230 & 236 & 242 & 248 & 254 & 3 \mathrm{MMin} & 306 & 312 & 318 & 324 & 330 & 336 & 342 & 348 & 354 & 4 \times 11\end{array}$

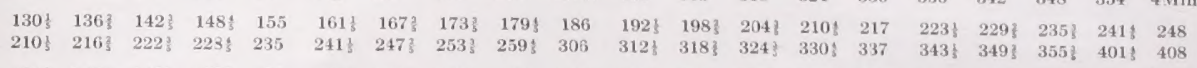

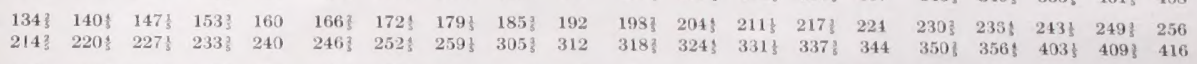

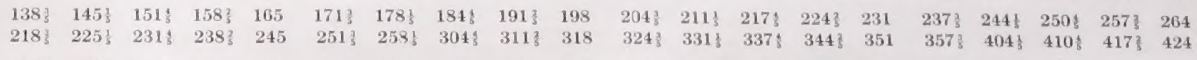

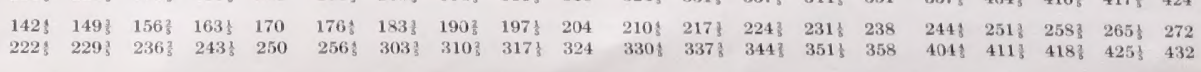

$\begin{array}{llllllllllllllllllll}147 & 154 & 161 & 168 & 175 & 182 & 189 & 196 & 203 & 210 & 217 & 224 & 231 & 238 & 245 & 252 & 259 & 266 & 273 & 280 \\ 227 & 234 & 241 & 248 & 255 & 302 & 309 & 316 & 323 & 330 & 337 & 344 & 351 & 358 & 405 & 412 & 419 & 426 & 433 & 440\end{array}$

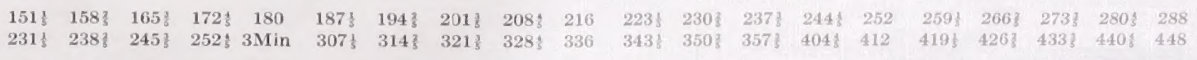

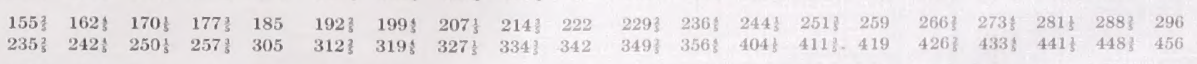

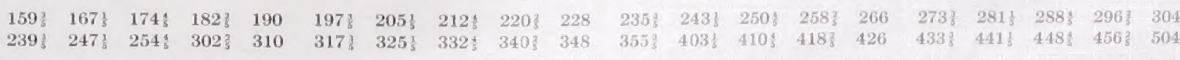

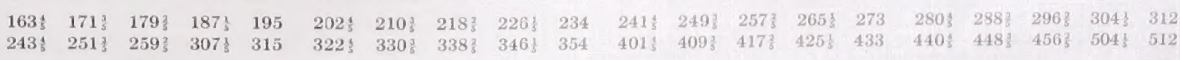

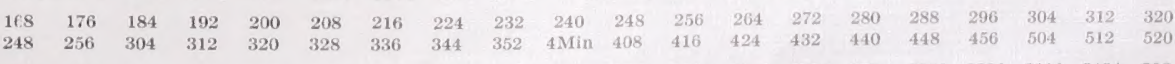

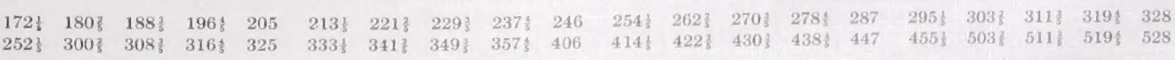

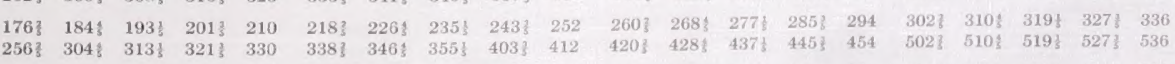

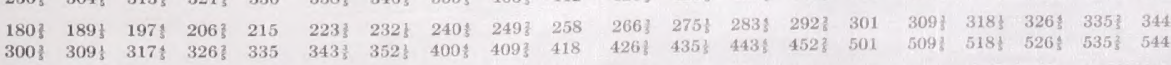

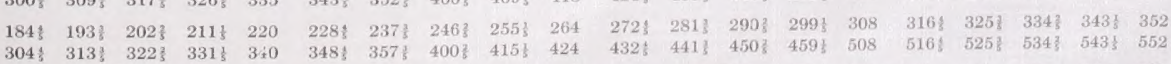
$\begin{array}{llllllllllllllllllll}189 & 198 & 207 & 216 & 225 & 234 & 243 & 252 & 261 & 270 & 279 & 288 & 297 & 306 & 315 & 324 & 333 & 342 & 351 & 360 \\ 309 & 318 & 327 & 336 & 345 & 354 & 403 & 412 & 421 & 430 & 439 & 448 & 457 & 506 & 515 & 524 & 533 & 542 & 551 & 6 \mathrm{Min} \\ 3 & & & & & & & & & & & & & & & \end{array}$ 





LIBRARY OF CONGRESS

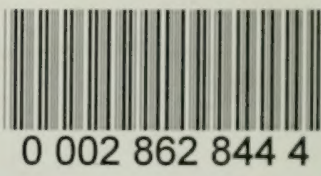

